Surveys in Differential Geometry, 2000

Vol. VII (C)2000, International Press

pp. $475-496$

\title{
MIRROR PRINCIPLE. IV
}

BONG H. LIAN, KEFENG LIU \& SHING-TUNG YAU

In honor of Professors M. Atiyah, R. Bott, F. Hirzebruch, and I. Singer.

\author{
Abstract \\ This is a continuation of Mirror Prenciple. III, [14].
}

\section{Some background}

This paper is a sequel to [12], [13], [14]. In this series of papers we develop mirror principle in increasing generality and breadth. Given a projective manifold $X$, mirror principle is a theory that yields relationships for and often computes the intersection numbers of cohomology classes of the form $b\left(V_{D}\right)$ on stable moduli spaces $\bar{M}_{g, k}(d, X)$. Here $V_{D}$ is a certain induced vector bundles on $\bar{M}_{g, k}(d, X)$ and $b$ is any given multiplicative cohomology class. In the first paper [12], we consider this problem in the genus zero $g=0$ case when $X=\mathbf{P}^{n}$ and $V_{D}$ is a bundle induced by any convex and/or concave bundle $V$ on $\mathbf{P}^{n}$. As a consequence, we have proved a mirror formula which computes the intersection numbers via a generating function. When $X=\mathbf{P}^{n}, V$ is a direct sum of positive line bundles on $\mathbf{P}^{n}$, and $b$ is the Euler class, a second proof of this special case has been given in [15], [2] following an approach proposed in [6]. Other proofs in this case has also been given in [1], and when $V$ includes negative line bundles, in [3]. In [13], we develop mirror principle when $X$ is a projective manifold with $T X$ convex. In [14], we consider the $g=0$ case when $X$ is an arbitrary projective manifold. Here emphasis has been put on a class of $T$-manifolds (which we call balloon manifolds) because in this case mirror principle yields a (linear!) reconstruction algorithm which computes in principle all the intersection numbers above for any convex/concave equivariant bundle 
$V$ on $X$ and any equivariant multiplicative class $b$. Moreover, specializing this theory to the case of line bundles on toric manifolds and $b$ to Euler class, we give a proof of the mirror formula for toric manifolds. In [14], we have also begun to develop mirror principle for higher genus. In this paper, we complete the theory for hight genus. We also extend the theory to include the intersection numbers for cohomology classes of the form $e v^{*}(\phi) b\left(V_{D}\right)$. Here $e v: \bar{M}_{g, k}(d, X) \rightarrow X^{k}$ is the usual evaluation map into the product $X^{k}$ of $k$ copies of $X$, and $\phi$ is any cohomology class on $X^{k}$.

For motivations and the main ideas of mirror principle, we refer the reader to the introduction of [12], [13].

Acknowledgement. B.H.L. wishes to thank the organizers for inviting him to lecture in the Conference on Geometry and Topology in honor of M. Atiyah, R. Bott, F. Hirzebruch, and I. Singer. Once again, we owe our special thanks to $\mathrm{J}$. $\mathrm{Li}$ who has been patiently lending his help to us throughout this project. B.H.L.'s research is supported by NSF grant DMS-0072158. K.L.'s research is supported by NSF grant DMS-9803234 and the Terman fellowship and the Sloane fellowship. S.T.Y.'s research is supported by DOE grant DE-FG02-88ER25065 and NSF grant DMS-9803347.

\section{Higher genus}

We assume that the reader is familiar with [14]. We follow the notations introduced there. Most results proved here have been summarized in Section 5.5 there.

In the first subsection, we give some examples of gluing sequences, a notion introduced in [14]. We also prove a quadratic identity, which is a generalization of Theorem 3.6 in [14] to higher genus plus multiple marked points. In the second subsection, we give a reconstruction algorithm which allows us to reconstruct the Euler series $A(t)$ associated to a gluing sequence in terms of Hodge integrals and some leading terms of $A(t)$.

1. Throughout this note, we abbreviate the data $(g, k ; d)$ as $D$ and write

$$
M_{D}=M_{g, k}\left((d, 1), X \times \mathbf{P}^{1}\right) .
$$

We denote by $L T_{D}(X), L T_{g, k}(d, X)$ the Li-Tian class of $M_{D}$ and $M_{g, k}(d, X)$ respectively.

2. In the last subsection, we prove the regularity of the collapsing 
map

$$
\varphi: M_{g, 0}\left((d, 1), \mathbf{P}^{n} \times \mathbf{P}^{1}\right) \rightarrow N_{d}
$$

generalizing Lemma 2.6 in [12]. Replacing $\mathbf{P}^{n}$ by the product

$$
Y=\mathbf{P}^{n_{1}} \times \cdots \times \mathbf{P}^{n_{m}}
$$

and $N_{d}$ by

$$
W_{d}=N_{d_{1}} \times \cdots \times N_{d_{m}}
$$

we get the map

$$
M_{g, 0}\left((d, 1), Y \times \mathbf{P}^{1}\right) \rightarrow W_{d} .
$$

Given an equivariant projective embedding $\tau: X \rightarrow Y$ with $A^{1}(X)$ $\cong A^{1}(Y)$ (see [14]), we have an induced map

$$
M_{D}=M_{g, k}\left((d, 1), X \times \mathbf{P}^{1}\right) \rightarrow M_{g, 0}\left((d, 1), Y \times \mathbf{P}^{1}\right) .
$$

Composing this with $M_{g, 0}\left((d, 1), Y \times \mathbf{P}^{1}\right) \rightarrow W_{d}$, we get a $G$-equivariant map

$$
M_{D} \rightarrow W_{d}
$$

which we also denoted by $\varphi$. This map will be used substantially to do functorial localization in the first subsection.

3. The standard $\mathbf{C}^{\times}$action on $\mathbf{P}^{1}$ induces an action on each $M_{D}$ (see section $5.5[14]$ ). The fixed point components are labelled by $F_{D_{1}, D_{2}}$ with $D_{1}=\left(g_{1}, k_{1} ; d_{1}\right), D_{2}=\left(g_{2}, k_{2} ; d_{2}\right), g_{1}+g_{2}=g, k_{1}+k_{2}=k$, $d_{1}+d_{2}=d$. As in the genus zero case, when $d_{1}, d_{2} \neq 0$, a stable map $\left(C, f, y_{1}, \ldots, y_{k}\right)$ in this component is given by gluing two pointed stable $\operatorname{maps}\left(f_{1}, C_{1}, y_{1}, . ., y_{k_{1}}, x_{1}\right) \in M_{g_{1}, k_{1}+1}\left(d_{1}, X\right),\left(f_{2}, C_{2}, y_{k_{1}+1}, \ldots, y_{k}, x_{2}\right) \in$ $M_{g_{2}, k_{2}+1}\left(d_{2}, X\right)$ with $f_{1}\left(x_{1}\right)=f_{2}\left(x_{2}\right)$, to $C_{0}=\mathbf{P}^{1}$ at 0 and $\infty$ at the marked points (cf. Section 3). We can therefore identify $F_{D_{1}, D_{2}}$ with $M_{g_{1}, k_{1}+1}\left(d_{1}, X\right) \times_{X} M_{g_{2}, k_{2}+1}\left(d_{2}, X\right)$. We also have a special component $F_{D, O}$ which is obtained by gluing a $k+1$ pointed stable map to $\mathbf{P}^{1}$ at either 0 , as described above. Likewise for $F_{O, D}$. We denote by

$$
i: F_{D_{1}, D_{2}} \rightarrow M_{D}
$$

the inclusions.

4. There are two obvious projection maps

$$
p_{0}: F_{D_{1}, D_{2}} \rightarrow M_{g_{1}, k_{1}+1}\left(d_{1}, X\right), \quad p_{\infty}: F_{D_{1}, D_{2}} \rightarrow M_{g_{2}, k_{2}+1}\left(d_{2}, X\right) .
$$

The map $p_{0}$ strips away (with the notations above) the stable maps $\left(f_{2}, C_{2}, y_{k_{1}+1}, \ldots, y_{k}, x_{2}\right)$ glued to the $\mathbf{P}^{1}$ at $\infty$, and forgets the $\mathbf{P}^{1} ; p_{\infty}$ 
strips away the stable map $\left(f_{1}, C_{1}, y_{1}, \ldots, y_{k_{1}}, x_{1}\right)$ glued to the $\mathbf{P}^{1}$ at 0 and forgets the $\mathbf{P}^{1}$.

5. We also have the following evaluation maps, and the forgetting map:

$$
\begin{aligned}
& e: F_{D_{1}, D_{2}} \rightarrow X, \quad e_{D}: M_{g, k+1}(d, X) \rightarrow X \\
& \rho: M_{g, k+1}(d, X) \rightarrow M_{g, k}(d, X) .
\end{aligned}
$$

Here $e$ evaluates a stable map in $F_{D_{1}, D_{2}}$ at the gluing point, $e_{D}$ evaluates a $k+1$ pointed stable map at the last marked point, and $\rho$ forgets the last marked point. Relating and summarizing the natural maps above is the following diagram:

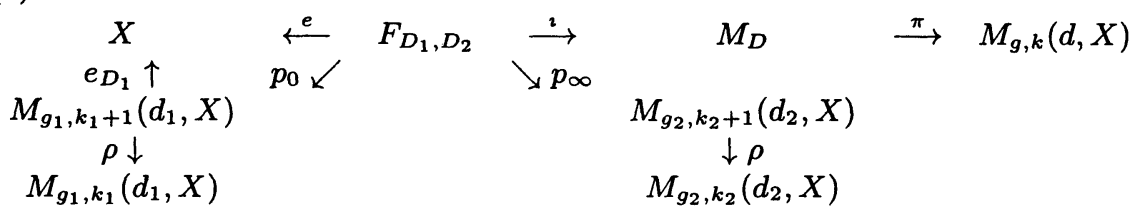

Here $\pi$ is natural morphism which maps $\left(C, f, y_{1}, . ., y_{k}\right) \in M_{D}$ to the stabilization of $\left(C, \pi_{1} \circ f, y_{1}, . ., y_{k}\right)$, where $\pi_{1}: X \times \mathbf{P}^{1} \rightarrow X$ is the projection. Note that we can identify $M_{g, k+1}(d, X)$ with $F_{O, D}$ via $p_{\infty}$. When $D_{1}=O:=(0,0 ; 0)$, the right part of the diagram above completes to a commutative triangle, i.e., $\pi \circ i=\rho$.

6. Let $\bar{M}_{g, k}$ be the Deligne-Mumford moduli space of $k$-pointed, genus $g$ stable curves. Recall the map

$$
M_{g, k}(d, X) \rightarrow \bar{M}_{g, k}
$$

which sends $\left(C, f, y_{1}, . ., y_{k}\right)$ to the stabilization of $\left(C, y_{1}, \ldots, y_{k}\right)$. Let $\mathcal{L}$ and $\mathcal{H}$ be respectively the universal line bundle and the Hodge bundle on $\bar{M}_{g, k+1}$. Thus $\mathcal{L}, \mathcal{H}$ have fibers at $\left(C, y_{1}, \ldots, y_{k}, x\right)$ given by $T_{x} C$ and $H^{0}\left(C, K_{C}\right) \cong H^{1}(C, \mathcal{O})^{*}$ respectively. We denote

$$
\Lambda_{g}(\xi)=\sum_{i=0}^{g} \xi^{\imath} c_{g-i}(\mathcal{H})
$$

for any formal variable $\xi$ (cf. [4]). We denote by the same notation the pullback of $\Lambda_{g}(\xi)$ to $M_{g, k+1}(d, X)$. We denote by $L_{D}$ the universal line bundle on $M_{g, k+1}(d, X)$. corresponding to the last marked point. 
7. Notations. In all formulas below involving $d, d_{1}, d_{2}$, and $g, g_{1}, g_{2}$, it is always assumed that

$$
\begin{aligned}
D & =(g, k ; d) \\
D_{1} & =\left(g_{1}, k_{1} ; d_{1}\right) \\
D_{2} & =\left(g_{2}, k_{2} ; d_{2}\right) \\
g & =g_{1}+g_{2} \\
k & =k_{1}+k_{2} \\
d & =d_{1}+d_{2} .
\end{aligned}
$$

Lemma 2.1 (cf. Lemma 3.5 [14]. ) Let $g=g_{1}+g_{2}, k_{1}+k_{2}=k$, $d=d_{1}+d_{2}$. For $d_{1}, d_{2} \neq 0$, the equivariant Euler class of the virtual normal bundle $N_{F_{D_{1}, D_{2}} / M_{D}}$ is

$$
\begin{aligned}
& e_{G}\left(F_{D_{1}, D_{2}} / M_{D}\right) \\
& \quad=p_{0}^{*}\left(\alpha\left(\alpha+c_{1}\left(L_{D_{1}}\right)\right) \Lambda_{g_{1}}(\alpha)^{-1}\right) p_{\infty}^{*}\left(\alpha\left(\alpha-c_{1}\left(L_{D_{2}}\right)\right) \Lambda_{g_{2}}(-\alpha)^{-1}\right) .
\end{aligned}
$$

For $d_{1}=0, g_{1}=0, k_{1}=0$,

$$
e_{G}\left(F_{O, D} / M_{D}\right)=-\alpha\left(-\alpha+c_{1}\left(L_{D}\right)\right) \Lambda_{g}(-\alpha)^{-1} .
$$

For $d_{2}=0, g_{2}=0, k_{2}=0$,

$$
e_{G}\left(F_{D, O} / M_{D}\right)=\alpha\left(\alpha+c_{1}\left(L_{D}\right)\right) \Lambda_{g}(\alpha)^{-1} .
$$

Proof. We consider the first equality, the other two being similar. We will compute the virtual normal bundle $N_{F_{D_{1}, D_{2}} / M_{D}}$ of $F_{D_{1}, D_{2}}$ in $M_{D}$ following the methods in [10], [7], [12], using the description of $F_{D_{1}, D_{2}}$ given above. We must identify the terms appearing in the tangent obstruction sequence of $M_{D}$. (See [7] Section 4.)

Consider the bundle $V:=\pi_{1}^{*} T X \oplus \pi_{2}^{*} T \mathbf{P}^{1}$ on $X \times \mathbf{P}^{1}$, where $\pi_{1}$ and $\pi_{2}$ are the projections from $X \times \mathbf{P}^{1}$ to $X$ and $\mathbf{P}^{1}$ respectively.

According to the description above, for each stable map $\left(C, f, x_{1}, \ldots, x_{k}\right)$ in $F_{D_{1}, D_{2}}$, we have an exact sequence over $C$ :

$$
0 \rightarrow f^{*} V \rightarrow f_{1}^{*} V \oplus f_{0}^{*} V \oplus f_{2}^{*} V \rightarrow V_{x_{1}} \oplus V_{x_{2}} \rightarrow 0 .
$$

Here $f_{0}$ is the restriction of $f$ to $C_{0}$, and $V_{x_{1}}, V_{x_{2}}$ denote respectively the bundles

$$
\pi_{1}^{*} T_{x} X \oplus \pi_{2}^{*} T_{0} \mathbf{P}^{1}, \quad \pi_{1}^{*} T_{x} X \oplus \pi_{2}^{*} T_{\infty} \mathbf{P}^{1}
$$


where $x=f_{0}\left(C_{0}\right) \in X$. From the long exact sequence associated to (8), we get the equality in the $\mathrm{K}$-group:

$$
\begin{aligned}
& H^{0}\left(C, f^{*} V\right)-H^{1}\left(C, f^{*} V\right) \\
& \quad=\sum_{\jmath=0}^{2}\left[H^{0}\left(C_{\jmath}, f_{j}^{*} V\right)-H^{1}\left(C_{j}, f_{\jmath}^{*} V\right)\right]-\left(V_{x_{1}}+V_{x_{2}}\right) .
\end{aligned}
$$

The tangent complex of $M_{D}$ restricted to $F_{D_{1}, D_{2}}$ is

$$
\begin{gathered}
H^{0}\left(C, f^{*} V\right)-H^{1}\left(C, f^{*} V\right)+T_{x_{1}} C_{1} \otimes T_{0} \mathbf{P}^{1} \\
+T_{x_{2}} C_{2} \otimes T_{\infty} \mathbf{P}^{1}-A_{C} \\
=\sum_{\jmath=0}^{2}\left[H^{0}\left(C_{j}, f_{j}^{*} V\right)-H^{1}\left(C_{\jmath}, f_{j}^{*} V\right)\right]-\left(V_{x_{1}}+V_{x_{2}}\right) \\
\quad+T_{x_{1}} C_{1} \otimes T_{0} \mathbf{P}^{1}+T_{x_{2}} C_{2} \otimes T_{\infty} \mathbf{P}^{1}-A_{C} .
\end{gathered}
$$

where $T_{x_{1}} C_{1} \otimes T_{0} \mathbf{P}^{1}$ and $T_{x_{2}} C_{2} \otimes T_{\infty} \mathbf{P}^{1}$ are contributions from the deformation of the nodes at $x_{1}, x_{2}$ of $C$, and $A_{C}$ is the contribution from the infinitesimal automorphisms of $C$. To get the moving parts of this, we subtract from it the fixed parts corresponding to $F_{D_{1}, D_{2}}=$ $M_{g_{1}, k_{1}+1}\left(d_{1}, X\right) \times_{X} M_{g_{2}, k_{2}+1}\left(d_{2}, X\right)$ (see description above). This is given by

$$
\sum_{j=1}^{2}\left[H^{0}\left(C_{j}, f_{j}^{*} \pi_{1}^{*} T X\right)-H^{1}\left(C_{\jmath}, f_{j}^{*} \pi_{1}^{*} T X\right)\right]-\pi_{1}^{*} T_{x} X-A_{C^{\prime}}
$$

where $C^{\prime}$ is the curve obtained from $C$ by contracting the component $C_{0}$. Note that here we have ignored the contributation coming from the deformation of $C_{1}, C_{2}$ in both (3) and (4), because the same contributation appear in both and hence this contributation cancels out in the difference.

We now compute and compare the terms in both (3) and (4) above. Since $f_{0}$ maps $C_{0}$ to a point $x$, we have

$$
\begin{aligned}
H^{1}\left(C_{0}, f_{0}^{*} \pi_{1}^{*} T X\right) & \simeq H^{1}\left(C_{0}, O\right) \otimes \pi_{1}^{*} T X=0 \\
H^{1}\left(C_{0}, f_{0}^{*} \pi_{2}^{*} T \mathbf{P}^{1}\right) & \simeq H^{1}\left(C_{0}, T C_{0}\right)=0 \\
H^{0}\left(C_{0}, f_{0}^{*} \pi_{1}^{*} T X\right) & =\pi_{1}^{*} T_{x} X .
\end{aligned}
$$


Similarly for $C_{1}$ and $f_{1}$, we have

$$
\begin{aligned}
H^{1}\left(C_{1}, f_{1}^{*} V\right) & =H^{1}\left(C_{1}, f_{1}^{*} \pi_{1}^{*} T X\right)+H^{1}\left(C_{1}, f_{1}^{*} \pi_{2}^{*} T \mathbf{P}^{1}\right) \\
H^{1}\left(C_{1}, f_{1}^{*} \pi_{2}^{*} T \mathbf{P}^{1}\right) & \simeq H^{1}\left(C_{1}, O\right) \otimes \pi_{2}^{*} T_{0} \mathbf{P}^{1} \\
H^{0}\left(C_{1}, f_{1}^{*} V\right) & =H^{0}\left(C_{1}, f_{1}^{*} \pi_{1}^{*} T X\right)+H^{0}\left(C_{1}, f_{1}^{*} \pi_{2}^{*} T \mathbf{P}^{1}\right) \\
H^{0}\left(C_{1}, f_{1}^{*} \pi_{2}^{*} T \mathbf{P}^{1}\right) & \simeq \pi_{2}^{*} T_{0} \mathbf{P}^{1} .
\end{aligned}
$$

Likewise, we have similar relations with $C_{1}, f_{1}, T_{0} \mathbf{P}^{1}$ replaced by $C_{2}, f_{2}, T_{\infty} \mathbf{P}^{1}$ everywhere.

Putting these formulas together, we get

$$
\begin{aligned}
N_{F_{D_{1}, D_{2}} / M_{D}}= & H^{0}\left(C_{0}, f_{0}^{*} \pi_{2}^{*} T \mathbf{P}^{1}\right)+T_{x_{1}} C_{1} \otimes T_{0} \mathbf{P}^{1} \\
& +T_{x_{2}} C_{2} \otimes T_{\infty} \mathbf{P}^{1}-H^{1}\left(C_{1}, O\right) \otimes \pi_{2}^{*} T_{0} \mathbf{P}^{1} \\
& -H^{1}\left(C_{2}, O\right) \otimes \pi_{2}^{*} T_{\infty} \mathbf{P}^{1}-A_{C_{0}}
\end{aligned}
$$

By taking equivariant Euler classes, we get the required formula. Here the terms $-H^{1}\left(C_{1}, O\right) \otimes \pi_{2}^{*} T_{0} \mathbf{P}^{1}$ and $-H^{1}\left(C_{2}, O\right) \otimes \pi_{2}^{*} T_{\infty} \mathbf{P}^{1}$ contribute $p_{0}^{*} \Lambda_{g_{1}}(\alpha)^{-1}$ and $p_{\infty}^{*} \Lambda_{g_{2}}(-\alpha)^{-1}$ respectively. The terms $T_{x_{1}} C_{1} \otimes T_{0} \mathbf{P}^{1}$ and $T_{x_{2}} C_{2} \otimes T_{\infty} \mathbf{P}^{1}$ contribute $\alpha+p_{0}^{*} c_{1}\left(L_{D_{1}}\right)$ and $-\alpha+p_{\infty}^{*} c_{1}\left(L_{D_{2}}\right)$ respectively, and the term $H^{0}\left(C_{0}, f_{0}^{*} \pi_{2}^{*} T \mathbf{P}^{1}\right)-A_{C_{0}}$ contributes $-\alpha^{2}$ (see [12, Section 2.3]).

Similarly, when $d_{1}=0, g_{1}=0, k_{1}=0$, we have

$$
\begin{aligned}
N_{F_{O, D} / M_{D}}= & H^{0}\left(C_{0}, f_{0}^{*} \pi_{2}^{*} T \mathbf{P}^{1}\right)+T_{x_{2}} C_{2} \otimes T_{\infty} \mathbf{P}^{1} \\
& -H^{1}\left(C_{2}, O\right) \otimes \pi_{2}^{*} T_{\infty} \mathbf{P}^{1}-A_{C_{0}}
\end{aligned}
$$

The term $-A_{C_{0}}$ contributes a factor $-\alpha$. Similarly for $d_{2}=0$ and $g_{2}=0$, we have

$$
\begin{aligned}
N_{F_{D, O} / M_{D}}= & H^{0}\left(C_{0}, f_{0}^{*} \pi_{2}^{*} T \mathbf{P}^{1}\right)+T_{x_{1}} C_{1} \otimes T_{0} \mathbf{P}^{1} \\
& -H^{1}\left(C_{1}, O\right) \otimes \pi_{2}^{*} T_{0} \mathbf{P}^{1}-A_{C_{0}}
\end{aligned}
$$

and now the term $-A_{C_{0}}$ contributes a factor $\alpha$. q.e.d.

\subsection{Gluing sequences}

Fix a class $\Omega \in A_{T}^{*}(X)$, such that $\Omega^{-1}$ is well-defined. We call the list of classes

$$
b_{D} \in A_{T}^{*}\left(M_{g, k}(d, X)\right) .
$$

an $\Omega$-gluing sequence if we have the (gluing) identities on the $F_{D_{1}, D_{2}}$ :

$$
e^{*} \Omega \cdot i^{*} \pi^{*} b_{D}=p_{0}^{*} \rho^{*} b_{D_{1}} \cdot p_{\infty}^{*} \rho^{*} b_{D_{2}}
$$


(This generalizes the definition in [14] to include the cases with multiple marked points.) Gluing sequences have the following obvious multiplicative property: that if $b_{D}$ and $b_{D}^{\prime}$ form two gluing sequences with respective to, say $\Omega$ and $\Omega^{\prime}$, then the product $b_{D} b_{D}^{\prime}$ form a gluing sequence with respective to $\Omega \Omega^{\prime}$.

Let $V$ be a $T$-equivariant bundle on $X$. Suppose that $H^{0}\left(C, f^{*} V\right)=0$ for every positive degree map $f: C \rightarrow X$ where $C$ a nonsingular genus $g$ curve. Then $V$ induces on each $M_{g, k}(d, X)$ a vector bundle $V_{D}$ whose fiber at $\left(C, f, y_{1}, . ., y_{k}\right)$ is $H^{1}\left(C, f^{*} V\right)$. We call such a $V$ a concave bundle on $X$.

Example 1. $X=\mathbf{P}^{n}$, and $V=\mathcal{O}(-k), k<0$.

Example 2. If $X$ is a projective manifold with $V=K_{X}<0$, then $V$ induces the bundles $V_{D}$. This is the situation in local mirror symmetry [12], [8].

Let $b_{T}$ be a $T$-equivariant multiplicative class. such that $\Omega=b_{T}(V)^{-1}$ is well-defined. Consider the classes $b_{T}\left(V_{D}\right) \in A_{T}^{*}\left(M_{g, k}(d, X)\right)$.

Lemma 2.2. The cohomology classes $b_{T}\left(V_{D}\right)$ form an $\Omega$-gluing sequence.

Proof. The proof is essentially the same as the argument for the genus zero gluing identity for a concave bundle $V$. See the first half of the proof of Theorem 3.6 [14]. q.e.d.

We now discuss a second example of a gluing sequence. Recall that a point $(f, C)$ in $F_{D_{1}, D_{2}}$ comes from gluing together a pair of stable maps $\left(f_{1}, C_{1}, y_{1}, . ., y_{k_{1}}, x_{1}\right),\left(f_{2}, C_{2}, y_{k_{1}+1}, . ., y_{k}, x_{2}\right)$ with $f_{1}\left(x_{1}\right)=f_{2}\left(x_{2}\right)$, to $C_{0}=\mathbf{P}^{1}$ at 0 and $\infty$, so that we have a long exact sequence

$$
\begin{aligned}
0 & \rightarrow H^{0}(C, \mathcal{O}) \rightarrow H^{0}\left(C_{1}, \mathcal{O}\right) \oplus H^{0}\left(C_{2}, \mathcal{O}\right) \rightarrow H^{0}\left(C_{0}, \mathcal{O}\right) \\
& \rightarrow H^{1}(C, \mathcal{O}) \rightarrow H^{1}\left(C_{1}, \mathcal{O}\right) \oplus H^{1}\left(C_{2}, \mathcal{O}\right) \rightarrow H^{1}\left(C_{0}, \mathcal{O}\right) \rightarrow 0
\end{aligned}
$$

Thus we have a natural isomorphism

$$
H^{1}(C, \mathcal{O}) \cong H^{1}\left(C_{1}, \mathcal{O}\right) \oplus H^{1}\left(C_{2}, \mathcal{O}\right) .
$$

This implies the isomorphism

$$
i^{*} \pi^{*} \mathcal{H}_{D}=p_{0}^{*} \rho^{*} \mathcal{H}_{D_{1}} \oplus p_{\infty}^{*} \rho^{*} \mathcal{H}_{D_{2}}
$$

of bundles on $F_{D_{1}, D_{2}}$. Here $\mathcal{H}_{D}$ denotes the bundle on $M_{g, k}(d, X)$ with fiber $H^{1}(C, \mathcal{O})$. (Note that for $g \geq 2$, then $\mathcal{H}_{D}$ is the pullback of the dual 
of the Hodge bundle $\mathcal{H}$ via the natural map $M_{g, k}(d, X) \rightarrow \bar{M}_{g, k}$.) Thus if $b$ is a multiplicative class, and $b_{D}:=b\left(\mathcal{H}_{D}\right)$, then the isomorphism above yields the gluing identity

$$
i^{*} \pi^{*} b_{D}=p_{0}^{*} \rho^{*} b_{D_{1}} \cdot p_{\infty}^{*} \rho^{*} b_{D_{2}}
$$

with $\Omega=1$. To summarize, we have

Lemma 2.3. The cohomology classes $b_{D}:=b\left(\mathcal{H}_{D}\right)$ above form a 1-gluing sequence.

Note that in both examples above, each class $b_{D}$ is naturally the pullback, via the forgetful map, of a class $b_{d}^{g} \in A_{T}^{*}\left(M_{g, 0}(d, X)\right)$. We will call a list of classes $b_{d}^{g} \in A_{T}^{*}\left(M_{g, 0}(d, X)\right)$ an $\Omega$-gluing sequence, if their pullbacks to $M_{g, k}(d, X)$ form a gluing sequence in the sense introduced above.

We now discuss a third construction. Fix a set of generators $\phi_{i}$ of $A_{T}^{*}(X)$, as a free module over $A_{T}^{*}(p t)$. Let

$$
\phi=\sum_{i} s_{i} \phi_{i}
$$

where $s_{i}$ are formal variables. Let

$$
\phi_{D}:=\prod_{j=1}^{k} e v_{j}^{*} \phi \in A_{T}^{*}\left(M_{g, k}(d, X)\right) .
$$

Here the map $e v_{j}$ evaluates at the $j$ th marked point of a stable map. Since the forgetting map $\rho$ commutes with evaluations, we have the identity

$$
i^{*} \pi^{*} \phi_{D}=p_{0}^{*} \rho^{*} \phi_{D_{1}} \cdot p_{\infty}^{*} \rho^{*} \phi_{D_{2}}
$$

Thus we have

Lemma 2.4. The cohomology classes $\phi_{D}$ above form a 1-gluing sequence.

Combining with the multiplicative property of gluing sequences, as explained above, this construction allows us to consider the intersection numbers of classes of the form $e v^{*}(\phi) b\left(V_{D}\right)$ on stable map moduli. In particular, this yields the GW-invariants twisted by a multiplicative class of the form $b\left(V_{D}\right)$. Here $e v$ is the evaluation map $M_{g, k}(d, X) \rightarrow X^{k}$ into the product of $k$ copies of $X$. The results below are easily generalized to the cases involving the additional factor $e v^{*}(\phi)$. 
For $\omega \in A_{G}^{*}\left(M_{D}\right)$, introduce the notation (cf. Section $3.2[14]$ )

$$
J_{D_{1}, D_{2}} \omega:=e_{*}\left(\frac{i^{*} \omega \cap\left[F_{D_{1}, D_{2}}\right]^{v i r}}{e_{G}\left(F_{D_{1}, D_{2}} / M_{D}\right)}\right) \in A_{*}^{T}(X)(\alpha) .
$$

Theorem 2.5 (cf. Theorem 3.6 [14]). Given a gluing sequence $b_{D} \in A_{T}^{*}\left(M_{g, k}(d, X)\right)$, we have the following identities in $A_{*}^{T}(X)(\alpha)$ :

$$
\Omega \cap J_{D_{1}, D_{2}} \pi^{*} b_{D}=\overline{J_{O, D_{1}} \pi^{*} b_{D_{1}}} \cdot J_{O, D_{2}} \pi^{*} b_{D_{2}} .
$$

Proof. Consider the fiber square

$$
\begin{array}{cccc}
F_{D_{1}, D_{2}} & \stackrel{\Delta^{\prime}}{\longrightarrow} & M_{g_{1}, k_{1}+1}\left(d_{1}, X\right) \times M_{g_{2}, k_{2}+1}\left(d_{2}, X\right) \\
e \downarrow & & & \downarrow e_{D_{1}} \times e_{D_{2}} \\
X & \stackrel{\Delta}{\longrightarrow} & X \times X
\end{array}
$$

where $\Delta$ is the diagonal inclusion. Recall also that (see Section 6 in [11])

$$
\left[F_{D_{1}, D_{2}}\right]^{v i r}=\Delta^{!}\left(L T_{g_{1}, k_{1}+1}\left(d_{1}, X\right) \times L T_{g_{2}, k_{2}+1}\left(d_{2}, X\right)\right) .
$$

Put

$$
\begin{aligned}
\omega=\frac{\rho^{*} b_{D_{1}}}{e_{G}\left(F_{D_{1}, O} / M_{D_{1}}\right)} \times \frac{\rho^{*} b_{D_{2}}}{e_{G}\left(F_{O, D_{2}} / M_{D_{2}}\right)} \\
\cap L T_{g_{1}, k_{1}+1}\left(d_{1}, X\right) \times L T_{g_{2}, k_{2}+1}\left(d_{2}, X\right) .
\end{aligned}
$$

From the fiber square (5), we have

$$
e_{*} \Delta^{!}(\omega)=\Delta^{*}\left(e_{D_{1}} \times e_{D_{2}}\right)_{*}(\omega)
$$

On the one hand is

$$
\begin{aligned}
\Delta^{*}\left(e_{D_{1}} \times e_{D_{2}}\right)_{*}(\omega)= & e_{D_{1 *}} \frac{\rho^{*} b_{D_{1}} \cap L T_{g_{1}, k_{1}+1}\left(d_{1}, X\right)}{e_{G}\left(F_{D_{1}, O} / M_{D_{1}}\right)} \\
& =\frac{\cdot e_{D_{2 *}} \frac{\rho^{*} b_{D_{2}} \cap L T_{g_{2}, k_{2}+1}\left(d_{2}, X\right)}{e_{G}\left(F_{O, D_{2}} / M_{D_{2}}\right)}}{J_{O, D_{1}} \pi^{*} b_{D_{1}}} \cdot J_{O, D_{2}} \pi^{*} b_{D_{2}} .
\end{aligned}
$$

Here we have use the fact that $\pi \circ i=\rho$. On the other hand, applying the gluing identity and Lemma (2.1), we have

$$
\begin{aligned}
e_{*} \Delta^{!}(\omega) & =e_{*}\left(p_{0}^{*} \frac{\rho^{*} b_{D_{1}}}{e_{G}\left(F_{D_{1}, O} / M_{D_{1}}\right)} \cdot p_{\infty}^{*} \frac{\rho^{*} b_{D_{2}}}{e_{G}\left(F_{O, D_{2}} / M_{D_{2}}\right)} \cap\left[F_{D_{1}, D_{2}}\right]^{v i r}\right) \\
& =e_{*}\left(\frac{e^{*} \Omega \cdot i^{*} \pi^{*} b_{D} \cap\left[F_{D_{1}, D_{2}}\right]^{v i r}}{e_{G}\left(F_{D_{1}, D_{2}} / M_{D}\right)}\right) \\
& =\Omega \cap J_{D_{1}, D_{2}} \pi^{*} b_{D} .
\end{aligned}
$$

This proves our assertion. q.e.d. 
Lemma 2.6 (cf. Lemma 3.2 [14]). Given a cohomology class $\omega$ on $M_{D}$, we have the following identities on the $\mathbf{C}^{\times}$fixed point component $Y_{d_{1}, d_{2}} \cong Y$ in $W_{d}$ :

$$
\frac{j^{*} \varphi_{*}\left(\omega \cap L T_{D}(X)\right)}{e_{G}\left(Y_{d_{1}, d_{2}} / W_{d}\right)}=\sum_{g_{1}+g_{2}=g, k_{1}+k_{2}=k} \tau_{*} e_{*}\left(\frac{i^{*} \omega \cap\left[F_{D_{1}, D_{2}}\right]^{v i r}}{e_{G}\left(F_{D_{1}, D_{2}} / M_{D}\right)}\right) .
$$

Proof. This follows from applying functorial localization to the diagram

$$
\begin{array}{cccc}
\left\{F_{D_{1}, D_{2}}\right\} & \stackrel{i}{\longrightarrow} & M_{D} & \\
\tau \circ e \downarrow & & \downarrow \varphi \\
Y_{d_{1}, d_{2}} & \stackrel{j}{\longrightarrow} & W_{d} . &
\end{array} \quad \text { q.e.d. }
$$

Now given a gluing sequence $b_{D}$, we put

$$
A_{D}:=J_{O, D} \pi^{*} b_{D}, \quad A_{d}:=\sum_{g, k} A_{D} \nu^{g} \mu^{k}, \quad A(t):=e^{-H \cdot t / \alpha} \sum_{d} A_{d} e^{d \cdot t} .
$$

Here $\nu, \mu$ are formal variables. Consider the class $\beta=\varphi_{*}\left(\pi^{*} b_{D} \cap L T_{D}(X)\right)$. We have

$$
\begin{aligned}
\int_{W_{d}} \beta \cap e^{\kappa \cdot \zeta} & =\sum_{d_{1}+d_{2}=d} \int_{Y_{d_{1}, d_{2}}} \frac{j^{*} \beta}{e_{G}\left(Y_{d_{1}, d_{2}} / W_{d}\right)} e^{\left(H+d_{1} \alpha\right) \cdot \zeta} \\
& =\sum_{D_{1}+D_{2}=D} \int_{Y_{d_{1}, d_{2}}} \tau_{*} J_{D_{1}, D_{2}} \pi^{*} b_{D} e^{\left(H+d_{1} \alpha\right) \cdot \zeta} \text { (Lemma 2.6) } \\
& =\sum_{D_{1}+D_{2}=D} \int_{X} J_{D_{1}, D_{2}} \pi^{*} b_{D} e^{\left(H+d_{1} \alpha\right) \cdot \zeta} \\
& =\sum_{D_{1}+D_{2}=D} \int_{X} \Omega^{-1} \cap \overline{A_{D_{1}}} \cdot A_{D_{2}} e^{\left(H+d_{1} \alpha\right) \cdot \zeta} \text { (Theorem 2.5). }
\end{aligned}
$$

Since $\beta \in A_{*}^{G}\left(W_{d}\right)$, hence $\int_{W_{d}} \beta \cap c \in A_{*}^{G}(p t)=\mathbf{C}\left[\mathcal{T}^{*}, \alpha\right]$ for all $c \in$ $A_{G}^{*}\left(W_{d}\right)$, it follows that both sides of the eqn. above lie in $\mathcal{R}[[\zeta]]$. Thus we get

Corollary 2.7. $A(t)$ is an Euler series.

We call $V$ be a $D$-critical concave bundle if the homogeneous degree of the class $b\left(V_{D}\right)$ is the same as the expected dimension of $M_{g, k}(d, X)$. We denote

$$
K_{D}=\int_{L T_{g, k}(d, X)} b\left(V_{D}\right)
$$


Lemma 2.8. If $V$ is a $D$-critical concave bundle, then in the $T$ nonequivariant limit we have the following formula

$$
\int_{X} e^{-H \cdot t / \alpha} J_{O, D} \pi^{*} b\left(V_{D}\right)=(-1)^{g} \alpha^{g-3}(2-2 g-k-d \cdot t) K_{D} .
$$

Proof. The integral above is equal to

$$
\begin{aligned}
\int_{L T_{g, k+1}(d, X)} e^{-e^{*} H \cdot t / \alpha} \frac{\rho^{*} b\left(V_{D}\right) \Lambda_{g}(-\alpha)}{\alpha(\alpha-c)} & \\
= & \int_{L T_{g, k}(d, X)} b\left(V_{D}\right) \Lambda_{g}(-\alpha) \rho_{*}\left(\frac{e^{-e^{*} H \cdot t / \alpha}}{\alpha(\alpha-c)}\right) .
\end{aligned}
$$

Here $\rho: M_{g, k+1}(d, X) \rightarrow M_{g, k}(d, X)$ forgets the last marked point.

Since the fiber of $\rho$ is of dimension 1, we take the degree 1 term in the fiber integral. The integration along the fiber $E$ is done in essentially the same way as in the genus zero case (see Theorem 3.12(ii) of [14]). It yields

$$
\int_{E} e^{*} H=d
$$

and

$$
\int_{E} c=2-2 g-k
$$

by Gauss-Bonnet formula. Since the degree of $b\left(V_{D}\right)$ coincides with the dimension of $L T_{g, k}(d, X)$, it follows that only the $\alpha^{0}$ term of $\Lambda_{g}(-\alpha)$ contributes to the integral above. q.e.d.

\subsection{Reconstruction}

From now on, we assume that $X$ is a balloon manifold, as in Sections 4.1 and 5.3 of [14]. We will find further constraints on a gluing sequence by computing the linking values of the Euler series $A(t)$. Recall that in genus zero, the linking values of an Euler series, say coming from $b_{T}\left(V_{d}\right)$, are determined by the restrictions $i_{F}^{*} b_{T}\left(\rho^{*} V_{d}\right)$ to the isolated fixed point $F=\left(\mathbf{P}^{1}, f_{\delta}, 0\right) \in M_{0,1}(d, X)$, which is a $\delta$-fold cover of a balloon $p q$ in $X$. In higher genus with multiple marked points, this will be replaced by a component in $M_{g, k+1}(d, X)$ consisting of the following stable maps $\left(C, f, y_{1}, . ., y_{k}, x\right)$. Here $C$ is a union of two curves $C_{1}$ and $C_{0} \cong \mathbf{P}^{1}$ such that $y_{1}, . ., y_{k} \in C_{1}$ and that $C_{0} \stackrel{f}{\rightarrow} p q$ is a $\delta$-fold cover with $f(x)=p, f\left(C_{1}\right)=q$. Therefore this fixed point component can be identified canonically with $\bar{M}_{g, k+1}$. For clarity, we will restrict the 
following discussion to the case of $k=0$. We'll denote the component by $F^{g}$. By convention, $F^{0}$ is the isolated fixed point $\left(\mathbf{P}^{1}, f_{\delta}, 0\right)$. Recall that (section 5.3 [14])

$$
e_{G}\left(F^{0} / M_{0,1}(d, X)\right)^{-1}=\frac{-\lambda}{\delta} \frac{e_{T}\left[H^{1}\left(\mathbf{P}^{1}, f_{\delta}^{*} T X\right)\right]^{\prime}}{e_{T}\left[H^{0}\left(\mathbf{P}^{1}, f_{\delta}^{*} T X\right)\right]^{\prime}} .
$$

Theorem 2.9. Suppose $g>0$. Let $p \in X^{T}, \omega \in A_{T}^{*}\left(M_{g, 1}(d, X)\right)[\alpha]$, and consider $i_{p}^{*} e_{*}\left(\frac{\omega \cap L T_{g, 1}(d, X)}{e_{G}\left(F_{O, D} / M_{D}\right)}\right) \in \mathbf{C}\left(\mathcal{T}^{*}\right)(\alpha)$ as a function of $\alpha$. Then

(i) Every possible pole of the function is a scalar multiple of a weight on $T_{p} X$.

(ii) Let $p q$ be a balloon in $X$, and $\lambda$ be the weight on the tangent line $T_{p}(p q)$. If $d=\delta[p q] \succ 0$, then the pole of the function at $\alpha=\lambda / \delta$ is of the form

$$
\begin{aligned}
& e_{T}(p / X) \frac{1}{\alpha(\alpha-\lambda / \delta)} \frac{1}{e_{T}\left(F^{0} / M_{0,1}(d, X)\right)} \\
& \cdot \int_{\left[F^{g}\right]^{v 2 r}} \frac{i_{F g}^{*} \omega \Lambda_{g}(\alpha) e_{T}\left(\mathcal{H}^{*} \otimes T_{q} X\right)}{\left(-\frac{\lambda}{\delta}+c_{1}(\mathcal{L})\right)} .
\end{aligned}
$$

Proof. The proof here is a slight modification of the genus zero case. We repeat the details here for the readers' convenience. Consider the commutative diagram

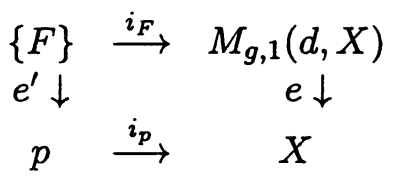

where $e$ is the evaluation map, $\{F\}$ are the fixed point components in $e^{-1}(p), e^{\prime}$ is the restriction of $e$ to $\{F\}$, and $i_{F}, i_{p}$ are the usual inclusions. By functorial localization we have, for any $\beta \in A_{T}^{*}\left(M_{g, 1}(d, X)\right)(\alpha)$,

$$
\begin{aligned}
i_{p}^{*} e_{*}\left(\beta \cap L T_{g, 1}(d, X)\right) & =e_{T}(p / X) \sum_{F} e_{*}^{\prime}\left(\frac{i_{F}^{*} \beta \cap[F]^{v i r}}{e_{T}\left(F / M_{g, 1}(d, X)\right)}\right) \\
& =e_{T}(p / X) \sum_{F} \int_{[F]^{v i r}} \frac{i_{F}^{*} \beta}{e_{T}\left(F / M_{g, 1}(d, X)\right)} .
\end{aligned}
$$

We apply this to the class

$$
\beta=\frac{\omega}{e_{G}\left(F_{O, D} / M_{D}\right)}=\frac{\omega \Lambda_{g}(-\alpha)}{\alpha(\alpha-c)}
$$


where $c=c_{1}\left(L_{D}\right)$ (cf. Lemma (2.1)). For (i), we will show that a pole of the sum (8) is at either $\alpha=0$ or $\alpha=\lambda^{\prime} / \delta^{\prime}$ for some tangent weight $\lambda^{\prime}$ on $T_{p} X$. For (ii), we will show that only one component $F$ in the sum (8) contributes to the pole at $\alpha=\lambda / \delta$, that the contributing component is $F^{g}$, and that the contribution has the desired form.

A fixed point $(C, f, x)$ in $e^{-1}(p)$ is such that $f(x)=p$, and that the image curve $f(C)$ lies in the union of the $T$-invariant balloons in $X$. The restriction of the first Chern class $c$ to an $F$ must be of the form

$$
i_{F}^{*} c=c_{F}+w_{F}
$$

where $c_{F} \in A^{1}(F)$, and $w_{F} \in \mathcal{T}^{*}$ is the weight of the representation on the line $T_{x} C$ induced by the linear map $d f_{x}: T_{x} C \rightarrow T_{p} X$. The image of $d f_{x}$ is either 0 or a tangent line $T_{p}(p r)$ of a balloon $p r$. Thus $w_{F}$ is either zero (when the branch $C_{1} \subset C$ containing $x$ is contracted), or $w_{F}=\lambda^{\prime} / \delta^{\prime}$ (when $C_{1} \stackrel{f}{\rightarrow} X$ maps by a $\delta^{\prime}$-fold cover of a balloon $p r$ with tangent weight $\left.\lambda^{\prime}\right)$. The class $e_{T}\left(F / M_{g, 1}(d, X)\right)$ is obviously independent of $\alpha$. Since $c_{F}$ is nilpotent, a pole of the sum (8) is either at $\alpha=0$ or $\alpha=w_{F}$ for some $F$. This proves (i).

Now, an $F$ in the sum (8) contributes to the pole at $\alpha=\lambda / \delta$ only if $w_{F}=\lambda / \delta$. Since the weights on $T_{p} X$ are pairwise linearly independent, that $\lambda / \delta=\lambda^{\prime} / \delta^{\prime}$ implies that $\lambda=\lambda^{\prime}$ and $\delta=\delta^{\prime}$. Since $d=\delta[p q]$, a fixed point $(C, f, x)$ contributing to the pole at $\alpha=\lambda / \delta$ must have the following form: that there is a branch $C_{0} \cong \mathbf{P}^{1}$ in $C$ such that $\left.f\right|_{C_{0}}: C_{0} \rightarrow p q$ is a $\delta$-fold cover with $f(x)=p$. Let $y \in C_{0}$ be the preimage of $q$ under this covering. Then the curve $C$ is a union of $C_{0}$ and a genus $g$ curve $C_{1}$ meeting $C_{0}$ at $y$, and $f\left(C_{1}\right)=q$. In other words, the fixed point component $F$ contributing to the pole at $\alpha=\lambda / \delta$ is $F^{g} \cong \bar{M}_{g, 1}$. It contributes to the sum (8) the term

$$
\int_{\left[F^{g}\right]^{v a r}} \frac{i_{F^{g}}^{*} \beta}{e_{T}\left(F^{g} / M_{0,1}(d, X)\right)}=\int_{F^{g}} \frac{i_{F^{g}}^{*} \omega \Lambda_{g}(-\alpha)}{\alpha\left(\alpha-\lambda / \delta-c_{F^{g}}\right)} \frac{1}{e_{T}\left(F^{g} / M_{g, 1}(d, X)\right)} .
$$

Here $c_{F^{g}} \in A^{1}\left(F^{g}\right)$ is zero because the universal line bundle $L_{d}^{g}$ restricted to $F^{g}$ is trivial (the line $T_{x} C$ is located at the marked point $x$ ).

We now compute the virtual normal bundle $N_{F^{g} / M_{0,1}(d, X)}$. A point $(C, f, x)$ in $F^{g}$ can be viewed as gluing two stable maps $\left(C_{0}, f_{0}, x, y\right) \in$ $M_{0,2}(d, X),\left(C_{1}, f_{1}, x_{1}\right) \in M_{g, 1}(0, X)$, by identifying $x_{1} \equiv y$. Here $f_{0}$ : $C_{0} \rightarrow p q$ is a $\delta$-fold cover with $f_{0}(x)=p, f_{0}(y)=q$, and $f_{1}\left(C_{1}\right)=q$. As 
before, we have

$$
\begin{aligned}
N_{F^{g} / M_{g, 1}(d, X)}= & {\left[H^{0}\left(C, f^{*} T X\right)\right]-\left[H^{1}\left(C, f^{*} T X\right)\right] } \\
& +\left[T_{y} C_{1} \otimes T_{y} C_{0}\right]+A_{C_{0}} \\
= & \left.\left(\left[H^{0}\left(C_{0}, f_{0}^{*} T X\right)\right]-H^{1}\left(C_{0}, f_{0}^{*} T X\right)\right]-A_{C_{0}}\right) \\
& -\left[H^{1}\left(C_{1}, f_{1}^{*} T X\right)\right]+\left[T_{y} C_{1} \otimes T_{y} C_{0}\right] .
\end{aligned}
$$

Note that the first three terms collected in the parentheses is the virtual normal bundle of $F^{0}$ in $M_{0,1}(d, X)$. The Euler class of this is constant on $F^{g}$, as given in eqn. (7). Since $f_{1}: C_{1} \rightarrow X$ maps to the point $q$, it follows that $\left[H^{1}\left(C_{1}, f_{1}^{*} T X\right)\right]=\mathcal{H}^{*} \otimes T_{q} X$ where $\mathcal{H}$ is the Hodge bundle on $\bar{M}_{g, 1}$. Clearly $\left[T_{y} C_{1} \otimes T_{y} C_{0}\right]=\mathcal{L} \otimes\left[\frac{-\lambda}{\delta}\right]$, where $\mathcal{L}$ is the universal line bundle on $\bar{M}_{g, 1}$, and $\left[\frac{-\lambda}{\delta}\right]$ is a 1 dimensional representation of that given weight. Therefore, we get

$$
e_{T}\left(F^{g} / M_{g, 1}(d, X)\right)=e_{T}\left(F^{0} / M_{0,1}(d, X)\right)\left(-\frac{\lambda}{\delta}+c_{1}(\mathcal{L})\right) e_{T}\left(\mathcal{H}^{*} \otimes T_{q} X\right)^{-1}
$$

Hence the contribution of the sum (8) to the pole at $\alpha=\lambda / \delta$ is

$$
\begin{aligned}
& e_{T}(p / X) \int_{\left[F^{g}\right]^{\text {vir }}} \frac{i_{F^{g}}^{*} \beta}{e_{T}\left(F^{g} / M_{0,1}(d, X)\right)} \\
&=e_{T}(p / X) \frac{1}{\alpha(\alpha-\lambda / \delta)} \frac{1}{e_{T}\left(F^{0} / M_{0,1}(d, X)\right)} \\
& \cdot \int_{\left[F^{g}\right]^{v \imath r}} \frac{i_{F^{g}}^{*} \omega \Lambda_{g}(-\alpha) e_{T}\left(\mathcal{H}^{*} \otimes T_{q} X\right)}{\left(-\frac{\lambda}{\delta}+c_{1}(\mathcal{L})\right)}
\end{aligned}
$$

This proves (ii). q.e.d.

Let $V$ be a concave bundle on $X$, and $b_{T}$ a choice of multiplicative class as before. Define the genus $g \geq 0$, degree $d=\delta[p q]$, linking values at the balloon $p q$ :

$$
L k_{g}:=e_{T}(p / X) \int_{\left[F^{g}\right]^{v i r}} \frac{i_{F^{g},}^{*} \beta}{e_{T}\left(F^{g} / M_{g, 1}(d, X)\right)}, \quad \beta:=\frac{\rho^{*} b_{T}\left(V_{D}\right)}{e_{G}\left(F_{O, D} / M_{D}\right)} .
$$

Corollary 2.10. For $g>0$,

$$
L k_{g}=L k_{0} \times \int_{\bar{M}_{g, 1}} \frac{b_{T}\left(\left.\mathcal{H}^{*} \otimes V\right|_{q}\right) \Lambda_{g}(-\alpha) e_{T}\left(\mathcal{H}^{*} \otimes T_{q} X\right)}{\left(-\frac{\lambda}{\delta}+c_{1}(\mathcal{L})\right)} .
$$

Proof. Restricting the bundle $\rho^{*} V_{D}$ on $M_{g, 1}(d, X)$ to the component $F^{g}$, we get

$$
i_{F^{g}}^{*} \rho^{*} V_{d}^{g}=\left[H^{1}\left(C, f^{*} V\right)\right]=\left[H^{1}\left(C_{0}, f_{0}^{*} V\right)\right] \oplus\left[H^{1}\left(C_{1}, f_{1}^{*} V\right)\right] .
$$


So, we have

$$
i_{F^{g}}^{*} \rho^{*} b_{T}\left(V_{D}\right)=i_{F^{0}}^{*} \rho^{*} b_{T}\left(V_{0,0 ; d}\right) b_{T}\left(\left.\mathcal{H}^{*} \otimes V\right|_{q}\right) .
$$

Again, the first factor is constant on $F^{g}$. Note that $F^{g}$ consists of orbifold points of order $\delta$. Thus the integral $\int_{\left[F^{g}\right]^{v i r}}$ can be written as $\frac{1}{\delta} \int_{\bar{M}_{g, 1}}$. Now applying the preceding theorem with $\omega=\rho^{*} b_{T}\left(V_{D}\right)$ yields the desired result. q.e.d.

In the special case $b_{T}=e_{T}$, the linking values become

$$
L k_{g}=L k_{0} \times \int_{\bar{M}_{g, 1}} \frac{\prod_{i} \Lambda_{g}\left(-\xi_{i}\right) \prod_{j} \Lambda_{g}\left(-\lambda_{j}\right) \Lambda_{g}(-\alpha)}{\left(-\frac{\lambda}{\delta}+c_{1}(\mathcal{L})\right)}
$$

where the $\xi_{i}$ and $\lambda_{j}$ are the weights on the isotropic representations $\left.V\right|_{q}$ and $T_{q} X$ respectively. These integral are nothing but Hodge integrals on $\bar{M}_{g, 1}$. Their values have been fully determined in [4].

Fix a concave bundle $V$ and multiplicative class $b_{T}$. Consider the associated Euler series $A(t)=e^{-H \cdot t / \alpha} \sum A_{D} \nu^{g} e^{d \cdot t}$ with coefficients

$$
A_{D}=e_{*}\left(\frac{\rho^{*} b_{T}\left(V_{D}\right) \cap L T_{g, 1}(d, X)}{e_{G}\left(F_{O, D} / M_{D}\right)}\right) \text {. }
$$

By Lemma (2.1), we see that

$$
\operatorname{deg}_{\alpha} A_{D} \leq-2+g
$$

Theorem 2.11. Consider the gluing sequence $b_{D}=\Lambda_{g}(\alpha)$ and suppose that $c_{1}(X)>0$. Then for a given $g$, the $A_{D}$ can be reconstructed from the linking values $L k_{g}$ and from finitely many degrees $d$.

Proof. Recall that the homology class $L T_{g, 1}(d, X)$ has dimension $s=$ exp.dim $M_{g, 1}(d, X)=(1-g)(\operatorname{dim} X-3)+\left\langle c_{1}(X), d\right\rangle+1$. Let $c=c_{1}\left(L_{D}\right)$. Then $c^{k} \cap L T_{g, 1}(d, X)$ is of dimension $s-k$, and so $e_{*}\left(c^{k} \cap L T_{g, 1}(d, X)\right)$ lies in the group $A_{s-k}^{T}(X)$. But this group is zero unless $s-k \leq \operatorname{dim} X$. The last condition means that

$$
-k+2 g \leq-\left\langle c_{1}(X), d\right\rangle+g(\operatorname{dim} X-1)+2 .
$$

For given $g$, the right hand side is negative for all but finitely many $d$. Suppose that $A_{D}$ are known for those finitely many $d$. Now

$$
\begin{aligned}
A_{D} & =\sum_{k \geq 0} \alpha^{-k-2} e_{*}\left(\Lambda(\alpha) \Lambda(-\alpha) c^{k} \cap L T_{g, 1}(d, X)\right) \\
& =\sum_{k \geq 0}(-1)^{g} \alpha^{-k-2+2 g} e_{*}\left(c^{k} \cap L T_{g, 1}(d, X)\right)
\end{aligned}
$$


So each of the unknown $A_{D}$ has order $\alpha^{-2+p}$. where $p<0$. By Theorem 4.3 [14], these $A_{D}$ can be reconstructed from the linking values. q.e.d.

The same argument shows that if $\left\{b_{D}\right\}$ is a given gluing sequence with the property that for a given $g$, the number

$$
-\left\langle c_{1}(X), d\right\rangle+g(\operatorname{dim} X-2)+2+\operatorname{deg} b_{D}
$$

is negative for all but finitely many $d$, then the theorem above holds for this gluing sequence.

\subsection{The collapsing lemma}

Let $X=\mathbf{P}^{1} \times \mathbf{P}^{n}$ and let $p_{1}, p_{2}$ be the first and the second projection of $\mathbf{P}^{1} \times \mathbf{P}^{n}$. We let $M_{g}(d, X)$ be the moduli space (stack) of stable morphisms from genus $g$ curves to $X$ of bi-degree $(1, d)$. The case $g=0$ was treated in [12]. Here we will prove a similar lemma in case $g \geq 1$. Note that there are no degree 1 maps from positive genus smooth curves to $\mathbf{P}^{1}$. Thus the domain of any stable morphism $f: C \rightarrow X$ in $M_{g}(d, X)$ must have a distinguished irreducible component $C_{0} \cong \mathbf{P}^{1}$ with

$$
\left.p_{1} \circ f\right|_{C_{0}}: C_{0} \stackrel{\sim}{\longrightarrow} \mathbf{P}^{1}
$$

and all other components mapping to points via $p_{1} \circ f$. Let $d_{0}$ be the degree of $\left.p_{1} \circ f\right|_{C_{0}}$. Use the collapsing map $M_{0}\left(d_{0}, X\right) \rightarrow N_{d_{0}}$, which depend on a choice of basis of $H^{0}\left(\mathcal{O}_{\mathbf{P}^{n}}(1)\right)$, we obtain $(n+1)$ sections

$$
\left[\phi_{0}, \cdots, \phi_{n}\right] \in H^{0}\left(\mathcal{O}_{\mathbf{P}^{1}}\left(d_{0}\right)\right)^{\oplus(n+1)} / \mathbf{C}^{\times} .
$$

Let $C_{1}, \cdots, C_{k}$ be other irreducible components of $C$ and let $z_{i} \in \mathbf{P}^{1}$ be $f\left(C_{i}\right)$ and $d_{i}$ be the degree of $f^{*} p_{2}^{*} \mathcal{O}_{\mathbf{P}^{n}}(1)$ over $C_{i}$. Note $d=\sum_{i=0}^{k} d_{i}$. Then using imbedding of sheaves

$$
\mathcal{O}_{\mathbf{P}^{1}}\left(d_{0}\right) \rightarrow \mathcal{O}_{\mathbf{P}^{1}}\left(\sum_{\imath=0}^{k} d_{i} z_{\imath}\right) \cong \mathcal{O}_{\mathbf{P}^{1}}(d)
$$

we obtain $(n+1)$-tuple of sections

$$
\left[\tilde{\phi}_{0}, \cdots, \tilde{\phi}_{n}\right] \in H^{0}\left(\mathcal{O}_{\mathbf{P}^{n}}(1)\right)^{\oplus(n+1)} / \mathbf{C}^{\times},
$$

which will be a point in $N_{d}$. This defines a correspondence

$$
\tilde{\varphi}: M_{g}(d, X) \longrightarrow N_{d} .
$$


Lemma 2.12. The correspondence $\tilde{\varphi}$ is induced by a morphism $\varphi$ : $M_{g}(d, X) \rightarrow N_{d}$. Moreover $\varphi$ is equivariant with respect to the induced action of $\mathbf{C}^{\times} \times T$.

Proof. The following proof is given by J. Li. Let $\mathcal{S}$ be the category of all schemes of finite type (over $\mathbf{C}$ ) and let $\mathcal{F}: \mathcal{S} \longrightarrow$ (Set) be the the contra-variant functor associating to each $S \in \mathcal{S}$ the set of families of stable morphisms

$$
F: \mathcal{X} \longrightarrow \mathbf{P}^{1} \times \mathbf{P}^{n} \times S
$$

over $S$ of bi-degree $(1, d)$ of arithmetic genus g, modulo the obvious equivalence relation. Note that $\mathcal{F}$ is represented by the moduli stack $M_{g}(d, X)$. Hence to define $\varphi$ it suffices to define a transformation

$$
\Psi: \mathcal{F} \longrightarrow \text { Mor }\left(-, N_{d}\right)
$$

We now define such a transformation. Let $S \in \mathcal{S}$ and let $\xi \in \mathcal{F}(S)$ be represented by $(\mathcal{X}, F)$. We let $p_{i}$ be the composite of $F$ with the $i$-th projection of $\mathbf{P}^{1} \times \mathbf{P}^{n} \times S$ and let $p_{i j}$ be the composite of $F$ with the projection from $\mathbf{P}^{1} \times \mathbf{P}^{n} \times S$ to the product of its $i$-th and $j$-th components. We consider the locally free sheaves $p_{2}^{*} \mathcal{O}_{\mathbf{P}^{n}}(k), k=0$ or 1 , of $\mathcal{O}_{\mathcal{X}}$-modules and its direct image complex

$$
\mathcal{L}_{\xi}(k)=R^{\bullet} p_{13 *} p_{2}^{*} \mathcal{O}_{\mathbf{P}^{n}}(k) .
$$

We claim that $\mathcal{L}_{\xi}(k)$, which is a complex of $\mathcal{O}_{\mathbf{P}^{1} \times S^{-}}$-modules, is quasiisomorphic to a perfect complex. Since this is a local problem, we can assume $S$ is affine. We pick a sufficiently relative-ample line bundle $H$ on $\mathcal{X} / S$ so that $p_{2}^{*} \mathcal{O}_{\mathbf{p}^{n}}(-k)$ is a quotient sheaf of

$$
\mathcal{V}_{1}=p_{3}^{*} p_{3 *}\left(\mathcal{O}(H) \otimes p_{2}^{*} \mathcal{O}_{\mathbf{P}^{n}}(-k)\right) \otimes \mathcal{O}(-H) .
$$

Let $\mathcal{V}_{2}$ be the kernel of $\mathcal{V}_{1} \rightarrow p_{2}^{*} \mathcal{O}_{\mathbf{p}^{n}}(-k)$. (Here $\mathcal{V}_{i}$ are implicitly depending on $k$.) Since $H$ is sufficiently ample, both $\mathcal{V}_{1}$ and $\mathcal{V}_{2}$ are locally free. Hence we have a short exact sequence of locally free sheaves of $\mathcal{O}_{\mathcal{X}}$-modules

$$
0 \longrightarrow p_{2}^{*} \mathcal{O}_{\mathbf{P}^{n}}(k) \longrightarrow \mathcal{V}_{1}^{\vee} \longrightarrow \mathcal{V}_{2}^{\vee} \longrightarrow 0
$$

We then apply $R^{\bullet} p_{13 *}$ to this exact sequence,

$$
0 \longrightarrow p_{13 *} p_{2}^{*} \mathcal{O}_{\mathbf{P}^{n}}(k) \longrightarrow p_{13 *} \mathcal{V}_{1}^{\vee} \longrightarrow p_{13 *} \mathcal{V}_{2}^{\vee} \longrightarrow R^{1} p_{13 *} p_{2}^{*} \mathcal{O}_{\mathbf{P}^{n}}(k) \longrightarrow 0
$$


Here all other terms vanish because $H$ is sufficiently ample and fibers of $p_{13}$ have dimension at most one. As argued in [12] both $p_{13 *} \mathcal{V}_{1}^{\vee}$ and $p_{13 *} \mathcal{V}_{2}^{\vee}$ are flat over $S$. Because $\mathbf{P}^{1} \times S \rightarrow S$ is smooth,

$$
p_{13 *} \mathcal{V}_{1}^{\vee} \longrightarrow p_{13 *} \mathcal{V}_{2}^{\vee}
$$

and hence $\mathcal{L}_{\xi}(k)$, is quasi-isomorphic to a perfect complex.

The complex $\mathcal{L}_{\xi}(k)$ satisfies the following base change property: let $\rho: T \rightarrow S$ be any base change and let $\rho^{*}(\xi) \in \mathcal{F}(T)$ be the pull back of $\xi$. Then there is a canonical isomorphism of complex of sheaves of $\mathcal{O}_{T}$-modules

$$
\mathcal{L}_{\rho^{*}(\xi)}(k) \cong\left(\mathbf{1}_{\mathbf{P}^{1}} \times \rho\right)^{*} \mathcal{L}_{\xi}(k) .
$$

Since $\mathcal{L}_{\xi}(k)$ is quasi-isomorphic to a perfect complex, we can define the determinant line bundle ${ }^{1}$ of $\mathcal{L}_{\xi}(k)$, denoted by $\operatorname{det} \mathcal{L}_{\xi}(k)$. It is an invertible sheaf of $\mathcal{O}_{\mathbf{P}^{1} \times S}$-modules. Using the Riemann-Roch theorem, one computes that the degree of $\operatorname{det}\left(\mathcal{L}_{\xi}(k)\right)$ along fibers of $\mathbf{P}^{1} \times S \rightarrow S$ are $k d-g$. Further, because $\mathcal{L}_{\xi}(k)$ has rank one, there is a canonical homomorphism

$$
\mathcal{L}_{\xi}(k) \longrightarrow \operatorname{det} \mathcal{L}_{\xi}(k)
$$

defined away from the support of the torsions subsheaves of $p_{13 *} p_{2}^{*} \mathcal{O}_{\mathbf{P}^{n}}(k)$ and $R^{1} p_{13 *} p_{2}^{*} \mathcal{O}_{\mathbf{P}^{n}}(k)$. Now let $w$ be any element in $H^{0}\left(\mathbf{P}^{n}, \mathcal{O}_{\mathbf{P}^{n}}(1)\right)$. Its pull back provides a canonical meromorphic section

$$
\sigma_{\xi, w} \in H^{0}\left(\mathbf{P}^{1} \times S, \mathbf{M}\left(\operatorname{det} \mathcal{L}_{\xi}(1)\right)\right) .
$$

For similar reason, the section $1 \in H^{0}\left(\mathcal{O}_{\mathbf{P}^{n}}\right)$ provides a canonical meromorphic section $\delta$ of $\operatorname{det} \mathcal{L}_{\xi}(0)$. Combined, they provide a canonical meromorphic section

$$
\eta_{\xi, w}=\sigma_{\xi, w} \cdot \delta^{-1} \in H^{0}\left(\mathbf{P}^{1} \times S, \mathbf{M}\left(\operatorname{det} \mathcal{L}_{\xi}(1) \otimes \operatorname{det} \mathcal{L}_{\xi}(0)^{-1}\right)\right) .
$$

We now show that $\eta_{\xi, w}$ extends to a regular section. Let $s \in S$ be any closed point. We first assume that there are no irreducible components of $\mathcal{X}_{s}$ that are mapped entirely to $\mathbf{P}^{1} \times w^{-1}(0) \subset \mathbf{P}^{1} \times \mathbf{P}^{n}$ under $F_{s}$. Here $F_{s}: \mathcal{X}_{s} \rightarrow \mathbf{P}^{1} \times \mathbf{P}^{n}$ is the restriction of $F$ to the fiber over $t \in S$. By shrinking $S$ if necessary, we can assume all $F_{t}: \mathcal{X}_{t} \rightarrow \mathbf{P}^{1} \times \mathbf{P}^{n}, t \in S$, have this property. Then the section $w$ induces a short exact sequence

$$
0 \longrightarrow \mathcal{O}_{\mathcal{X}} \longrightarrow p_{2}^{*} \mathcal{O}_{\mathbf{P}^{n}}(1) \longrightarrow \mathcal{R} \longrightarrow 0
$$

\footnotetext{
${ }^{1}$ All materials concerning determinant line bundle are taken from [9].
} 
and a long exact sequence

$\longrightarrow R^{\bullet} p_{13 *} \mathcal{O}_{\mathcal{X}} \longrightarrow R^{\bullet} p_{13 *} p_{2}^{*} \mathcal{O}_{\mathbf{P}^{n}}(1) \longrightarrow R^{\bullet} p_{13 *} \mathcal{R} \longrightarrow R^{\bullet+1} p_{13 *} \mathcal{O}_{\mathcal{X}} \longrightarrow$

By our assumption on $w, R^{1} p_{13 *} \mathcal{R}=0$. Next, we let $\mathcal{E}_{1} \rightarrow \mathcal{E}_{2}$ (resp. $\mathcal{F}_{1} \rightarrow \mathcal{F}_{2}$ ) be the complex (9) associated to $k=1$ (resp. $k=0$ ). Clearly, we have canonical commutative diagram

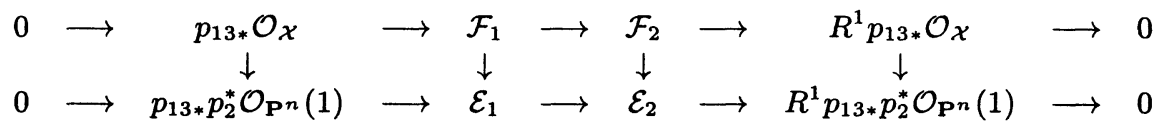

of short exact sequences. Let

$$
\mathcal{K}_{1}: \mathcal{F}_{1} \longrightarrow \mathcal{E}_{1} \oplus \mathcal{F}_{2} \longrightarrow \mathcal{E}_{2}
$$

be the induced complex. Note the last arrow is surjective. Let $\mathcal{A}_{1}$ be $\mathcal{F}_{1}$ and $\mathcal{A}_{2}$ be the kernel of the last arrow of the above complex. Hence $\mathcal{K}_{1}$ is quasi-isomorphic to the complex

$$
\mathcal{K}_{2}: \quad \mathcal{A}_{1} \longrightarrow \mathcal{A}_{2}
$$

Note that both $\mathcal{A}_{1}$ and $\mathcal{A}_{2}$ are $\mathcal{O}_{S}$-flat. Hence we can define the determinant line bundle $\operatorname{det} \mathcal{K}_{2}$. We then have canonical isomorphisms

$$
\operatorname{det} \mathcal{K}_{2} \cong \operatorname{det} \mathcal{K}_{1} \cong \operatorname{det} \mathcal{L}_{\xi}(1)^{-1} \otimes \operatorname{det} \mathcal{L}_{\xi}(0)
$$

Now let $t \in S$ be any closed point. $t \in S$. It is clear that the restriction of (10) to general points of $\mathbf{P}_{t}^{1}$ is an isomorphism. Hence $\operatorname{det} \mathcal{K}_{2}^{-1}$ has a canonical section over $\mathbf{P}^{1} \times S$ [5]. It is direct to check that under the isomorphism (11) this section is the extension of $\eta_{\xi, w}$. Since $\mathbf{P}^{1} \times S \rightarrow S$ is proper, such extension is unique.

It remains to show that $\eta_{\xi, w}$ can be extended even the assumption on $w^{-1}(0)$ does not hold. Note that in this case, we can find two sections $w_{1}$ and $w_{2}$ in $H^{0}\left(\mathcal{O}_{\mathbf{P}^{n}}(1)\right)$ so that $w=w_{1}+w_{2}$ and that both $w_{1}$ and $w_{2}$ satisfies the condition about $w_{1}^{-1}(0)$ and $w_{2}^{-1}(0)$. Here we might need to shrink $S$ if necessary. Then $\eta_{\xi, w_{1}}$ and $\eta_{\xi, w_{2}}$ both can be extended to regular sections in

$$
H^{0}\left(\mathbf{P}^{1} \times S, \operatorname{det} \mathcal{L}_{\xi}(1) \otimes \operatorname{det} \mathcal{L}_{\xi}(0)^{-1}\right)
$$

Further, over the open subset $Z \subset \mathbf{P}^{1} \times S$ where all $R^{i} p_{13 *} p_{2}^{*} \mathcal{O}_{\mathbf{P}^{n}}(k)$, $i, k=0,1$, are torsion free, we obviously have

$$
\eta_{\xi, w}=\eta_{\xi, w_{1}}+\eta_{\xi, w_{2}}
$$


Since $Z \cap \mathbf{P}^{1} \times\{t\} \neq \emptyset$ for all $t \in S$, the right hand side of the above identity provides an extension of $\eta_{\xi, w}$. This proves that for any $w \in$ $H^{0}\left(\mathcal{O}_{\mathbf{P}^{n}}(1)\right)$ the meromorphic sections $\eta_{\xi, w}$ extends to a regular section

$$
\eta_{\xi, w}^{\mathrm{ex}} \in H^{0}\left(\mathbf{P}^{1} \times S, \operatorname{det} \mathcal{L}_{\xi}(1) \otimes \operatorname{det} \mathcal{L}_{\xi}(0)^{-1}\right) .
$$

Again since $\mathbf{P}^{1} \times S \rightarrow S$ is smooth and proper, the extension is unique.

Now we define the morphism $S \rightarrow N_{d}$. Let $\left\{w_{0}, \cdots, w_{n}\right\}$ be a basis of $H^{0}\left(\mathcal{O}_{\mathbf{P}^{n}}(1)\right)$. Then we obtain $(n+1)$ canonical regular sections

$$
\eta_{\xi, w_{0}}^{\mathrm{ex}}, \cdots, \eta_{\xi, w_{n}}^{\mathrm{ex}} \in H^{0}\left(\mathbf{P}^{1} \times S, \operatorname{det} \mathcal{L}_{\xi}(1) \otimes \operatorname{det} \mathcal{L}_{\xi}(0)^{-1}\right) .
$$

Hence, after fixing an isomorphism

$$
\operatorname{det} \mathcal{L}_{\xi}(1) \otimes \operatorname{det} \mathcal{L}_{\xi}(0)^{-1} \cong \pi_{S}^{*} \mathcal{M} \otimes \pi_{\mathbf{P}^{1}}^{*} \mathcal{O}_{\mathbf{P}^{1}}(d)
$$

for some invertible sheaf $\mathcal{M}$ of $\mathcal{O}_{S}$-modules, we obtain $(n+1)$ canonical sections of

$$
H^{0}\left(\mathcal{O}_{\mathbf{P}^{1}}(d)\right) \otimes_{\mathbf{C}} \mathcal{M}
$$

which defines a morphism

$$
S \longrightarrow H^{0}\left(\mathcal{O}_{\mathbf{P}^{n}}(1)\right)^{\oplus(n+1)} / \mathbf{C}^{*} .
$$

Since the image is always away from 0 , it defines a morphism

$$
S \longrightarrow N_{d}
$$

It is routine to check that this construction satisfies the base change property, and hence defines a morphism $M_{g}(d, X) \rightarrow N_{d}$, as desired.

To check that this morphism gives rise to the correspondence mentioned before, it suffices to check the case where $S$ is a closed point. In this case one sees immediately that the complex $\mathcal{L}_{\xi}(1)-\mathcal{L}_{\xi}(0)$ has locally free part isomorphic to $\mathcal{O}_{\mathbf{P}^{1}}\left(d_{0}\right)-\mathcal{O}_{\mathbf{P}^{1}}$, and has torsion part supported at $z_{\imath}$ of length $d_{\imath}$. Further, a direct check shows that the sections $\sigma_{\xi, w_{i}} \cdot \delta^{-1}$ are a non-zero constant multiple of the sections $\tilde{\phi}_{i}$ mentioned in the definition of the correspondence. This shows that the morphism defines the correspondence constructed. The equivariant property of this morphism again follows from the base change property of this construction. This completes the proof of the Collapsing Lemma. q.e.d. 


\section{References}

[1] A. Bertram, Another way to enumerate rational curves with torus action, math. AG/9905159.

[2] G. Bini, C. De Concini, M. Polito \& C. Procesi, Givental's work relative to mirror symmetry, math.AG/9805097.

[3] A. Elezi, Mirror symmetry for concavex bundles on projective spaces, math. AG/0004157.

[4] C. Faber \& R. Pandharipande, Hodge integrals and Gromov-Witten theory, math. AG/9810173.

[5] R. Friedman, J. W. Morgan, Smooth four-manifolds and complex surfaces, Ergebnisse der Mathematik und ihrer Grenzgebiete (3), Results in Mathematics and Related Areas (3), Springer, Berlin, Vol. 27, 1994.

[6] A. Givental, Equivariant Gromov-Witten invariants, alg-geom/9603021.

[7] T. Graber \& R. Pandharipande, Localization of virtual classes, alg-geom/9708001.

[8] S. Katz, A. Klemm \& C. Vafa, Geometric engineering of quantum field theories, Nucl. Phys. B497 (1997) 173-195.

[9] F. F. Knudsen \& D. Mumford, The projectivity of the moduli space of stable curves. I. Preliminaries on "det" and "Div", Math. Scand. 39 (1976) 19-55.

[10] M. Kontsevich, Enumeration of rational curves via torus actions, The Moduli Space of Curves, (eds. R. Dijkgraaf, C. Faber, G. van der Geer), Progr. Math., Birkhäuser, Vol. 129, 1995, 335-368.

[11] J. Li \& G. Tian, Virtual moduli cycle and Gromov-Witten invariants of algebraic varieties, J. Amer. Math. Soc. 11 (1998) 119-174.

[12] B. Lian, K. Liu \& S.T. Yau, Mirror principle. I, Asian J. Math. 1 (1997) 729-763.

[13] — Mirror principle. II, Asian J. Math. 3 (1999) ???-???.

[14] - Mirror principle. III, math.AG/9912038.

[15] R. Pandharipande, Rational curves on hypersurfaces (after givental), math. AG/9806133.

BRANDEIS UNIVERSITY

STANFORD UNIVERSITY

HARVARD UNIVERSITY 